Cascade Totals: Youth Friendly Services data for October 2007 to January 2011

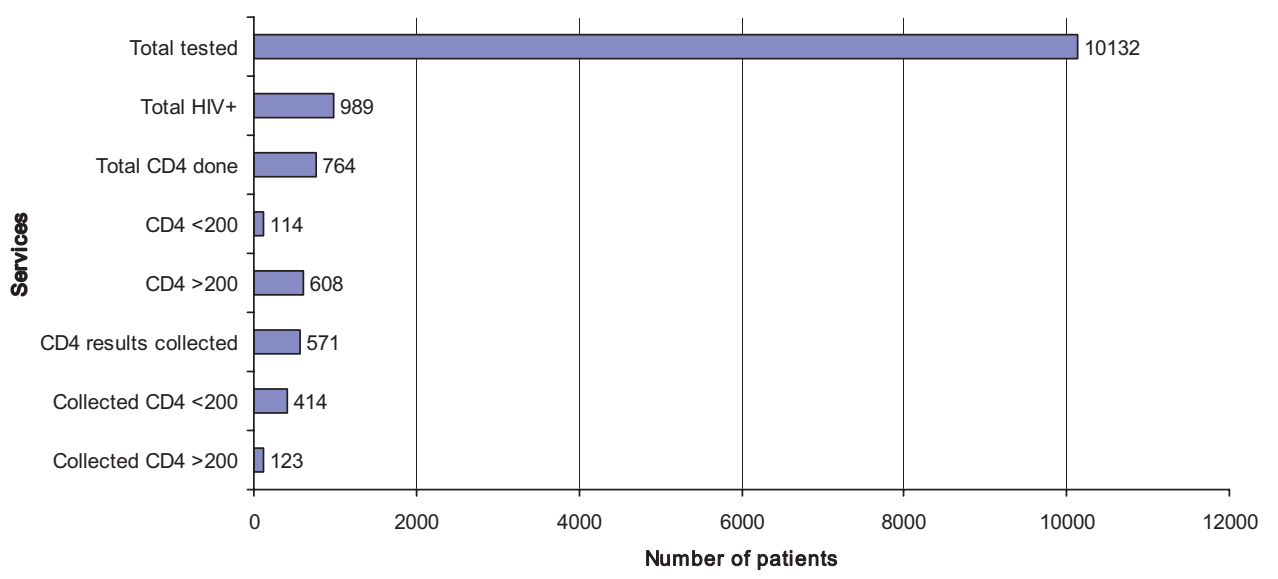

Abstract P1-S6.38 Figure 1 Cascade of HIV service bundling for October 2007-January 2011.

counsellors for HIV and STI testing who are skilled in working with youth. This abstract discusses the HIV and STI outputs of the Youth Friendly clinic based on the quantitative reported data.

Methods Routine programme data submitted for the period October 2007-January 2011 was reviewed. Data was collated for the purposes of graphing, and calculating percentage difference and percentage change.

Results The target age for service delivery fluctuated in line with WHO and is currently 15-24 years. There was inconsistent reporting of headcounts for the review period. In 2007 the clinic saw 15-19 year olds (ration of 1 male: 2 female). From 2008 this changed to a ratio of $30 \%$ male: $70 \%$ female. The age threshold moved to 24 years and clinic population became $35 \%$ of $15-19$ year olds: $65 \%$ of 20-24 year olds. Total number of HIV tests conducted was 10132 (3515 males and 6617 females). 10\% tested HIV positive. 77\% $(\mathrm{n}=764)$ had blood draws for CD4 count testing. 722 results were received from the laboratory, of which 571 (79\%) were collected by patients. 414 results were $<200$ cells $/ \mathrm{mm}^{3}$ see Abstract P1-S6.38 Figure 1. All eligible patients were worked up for ARVs and referred to the initiation facilities. 5176 clients accessed STI services. In 2007 $50 \%$ males and $50 \%$ females were treated for STIs. During the review period this changed to $44 \%$ males and $56 \%$ females. Both HIV testing and STI services were lowest from October 2007 to February 2008 and peaked from May 2009-to July 2009 and again from September 2009 to November 2009. The reported data did not distinguish between new and repeat clients for HIV testing and STI treatment. Conclusion The increase in target population age led to more services being accessed by 20-24 year olds. Over time there was an increase in the number of people accessing HIV and STI testing and treatment. There was an increase in males accessing the clinic and a decrease in STI services required by males. Lab reporting of CD4 counts and CD4 results collection needs to be strengthened. Routing data needs to be collected on new and repeat clients as a means of addressing casual factors for STI acquisition.

\section{P1-S6.39 CAN PROFESSIONAL MIDWIVES INTERPRET RAPID SYPHILIS TESTS ACCURATELY?}

doi:10.1136/sextrans-2011-050108.263

${ }^{1} \mathrm{M}$ A Chiappe Gutiérrez, ${ }^{1} \mathrm{~K}$ Soto, ${ }^{1} \mathrm{~L}$ Lopez-Torres, ${ }^{1} \mathrm{C}$ Cárcamo, ${ }^{1} \mathrm{M}$ I Valderrama Calderón, ${ }^{1} \mathrm{~S}$ La Rosa, ${ }^{1} \mathrm{P}$ Mallma, ${ }^{1} \mathrm{P}$ Garcia, ${ }^{2} \mathrm{R}$ Peeling. ${ }^{1}$ School of Public Health and Administration, Universidad Peruana Cayetano Heredia, Lima, Peru; ${ }^{2}$ London School of Hygiene \& Tropical Medicine, UK

Background Congenital syphilis produces miscarriage and severe complications in newborns. Rapid syphilis testing (RST, Syphilis 3.0
Bioline) was introduced in antenatal care and reproductive health services of 16 health centers at Ventanilla (Callao), Peru, aiming to improving access to syphilis screening and treatment in the first contact of pregnant women with professional midwives. We present the results of a quality control assessment evaluating internal (IOC) and external quality control (EOC) of such tests when performed by professional midwives.

Methods We developed quality controls panels consisting of the Dried Tube Specimen (DTS). Open label positive and a negative control DTS were distributed as IOC bimonthly to services performing RST, to evaluate the test performance under the service storage conditions. Additionally, closed label controls (EOC) were distributed biyearly to evaluate the performance of each professional midwife performing RST. The ECC panel was composed of a weak positive, an intermediate and a strong positive DTS, as well as a negative DTS. Midwives were trained in DTS reconstitution, as well as result interpretation and recording.

Results 28 DTS panels were distributed for IOC (16 to antenatal and 3 to emergency services), with 100\% concordance. 119 DTS panels were distributed as EQC, with 100\% concordance observed in most $(95 \%)$ of midwifes evaluated. Six midwives read the weak positive DTS as negative ( $75 \%$ concordance). These six professionals were retrained in their health centers. IOC and EQC were well accepted by midwifes, giving them a sense of confidence with their performance with the test.

Conclusion Excellent IQC and EQC results were observed, with good acceptance of the evaluation by midwives.

\section{P1-S6.40 VCT SITES IN MSIM FROM MEXICO CITY: AN OPPORTUNITY TO ACTION}

doi:10.1136/sextrans-2011-050108.264

${ }^{1} \mathrm{C}$ Magis-Rodríguez, ${ }^{1} \mathrm{~A}$ González-Rodríguez, E Bravo-García, 'L Juárez-Figuroa, ${ }^{1}$ E Rodríguez-Nolasco, ${ }^{1} \mathrm{C}$ De Azcárraga Urteaga. ${ }^{1}$ Programme on HIVIAIDS in Mexico City, Mexico, Mexico

Background Mexico has a concentrated HIV epidemic (15\% HIV prevalence among Male Sex Workers; $11 \%$ in MSM; $5 \%$ in IDU; and $2 \%$ in FSW). Voluntary counselling and testing (VCT) for HIV is a strategy for case detection and deliver prevention messages. This study analyses the HIV prevalence in MSM in two different settings, in order to identify potential opportunities for HIV prevention.

Methods We realised a comparative study between the men that received VCT inside the clinic, and the men reached with VCT in a mobile unit. Both services were offered by the Clinica Condesa in 